\title{
Deep neck abscess: an analysis of microbial etiology and the effectiveness of antibiotics
}

\author{
Shih-Wei Yangl,2 \\ Ming-Hsun Lee L,4 $^{3,4}$ \\ Lai-Chu See ${ }^{5}$ \\ Shu-Huan Huang ${ }^{6}$ \\ Tsung-Ming Chen ${ }^{7}$ \\ Tai-An Chen ${ }^{1,4}$ \\ 'Department of Otolaryngology- \\ Head and Neck Surgery, Chang \\ Gung Memorial Hospital, Keelung; \\ ${ }^{2}$ Graduate Institute of Clinical \\ Medical Sciences, Chang Gung \\ University, Taoyuan; ${ }^{3}$ Division of \\ Infectious Diseases, Department \\ of Internal Medicine, Chang Gung \\ Memorial Hospital, Linkou; ${ }^{4}$ School \\ of Medicine, Chang Gung University, \\ Taoyuan; ${ }^{5}$ Biostatistics Consulting \\ Center, Department of Public Health, \\ Chang Gung University, Taoyuan; \\ ${ }^{6}$ Department of Pathology, Chang \\ Gung Memorial Hospital, Keelung; \\ ${ }^{7}$ Department of Otolaryngology-Head \\ and Neck Surgery, Koo Foundation \\ Sun Yet-Sen Cancer Center, Taipei, \\ Taiwan
}

Correspondence: Shih-Wei Yang Department of Otolaryngology-Head and Neck Surgery, Chang Gung Memorial Hospital, Keelung. No 222 Mai Chin Road, Keelung city, 204, Taiwan

Email sweeyang@gmail.com

Tel +8862 243। 3। 3I ext 2445

$\mathrm{Fax}+886224313161$

\begin{abstract}
The objective was to demonstrate the aerobic and anaerobic microbiology of deep neck space abscess and to analyze the coverage rate of different empiric antimicrobial agents. A retrospective review of hospitalized patients with deep neck abscess diagnosed at a tertiary-care, general hospital between April 2001 and October 2006. The study enrolled 100 patients. The bacterial cultures of 89 patients yielded positive results (89\%). The predominant aerobes were viridans streptococci, Klebsiella pneumoniae, and Staphylococcus aureus. The predominant anaerobes included species of Prevotella, Peptostreptococcus, and Bacteroides. Five different combinations of empiric antibiotics, namely regimen 1: penicillin $\mathrm{G}$ and clindamycin and gentamicin, regimen 2: ceftriaxone and clindamycin, regimen 3: ceftriaxone and metronidazole, regimen 4: cefuroxime and clindamycin, and regimen 5: penicillin and metronidazole, were compared using the antimicrobial susceptibility of 89 cases. The coverage rates of regimens $1,2,3,4$, and 5 were $67.4 \%, 76.4 \%, 70.8 \%, 61.8 \%$, and $16.9 \%$, respectively. The coverage of regimen 5 was considerably worse than that of the other four regimens $(p<0.001)$. Regimen 2 was significantly better than regimen $4(p<0.001)$. Regimen 2 had better coverage than regimens $1(\mathrm{p}=0.096)$ and $3(\mathrm{p}=0.302)$, but the difference was not statistically significant. This study demonstrates the bacteriology of deep neck abscess and analyzes the coverage rate of different empiric antimicrobial agents. Regimens 1, 2, and 3 could be good candidates for empiric antibiotics. Pathogen-directed antimicrobial therapy should be adjusted after the culture results are obtained.
\end{abstract}

Keywords: deep neck abscess, microbiology, antibiotics, empiric

\section{Introduction}

Deep neck abscesses are less common today than in the past. The impact of antibiotic treatment and improved dental care are the most likely reasons for this change. In spite of widespread use of antibiotics, deep neck infections do not disappear and remain one of the difficult emergencies encountered in daily clinical practice. Once an abscess occupies one of the deep neck spaces, the infection can spread across the spaces or damage the adjacent vital neural or vascular structures. The extent and severity of the illness could become life threatening. In addition to the systemic toxicity and localized respiratory and digestive tract disturbance, more serious complications, including airway obstruction, pneumonia, lung abscess, mediastinitis, pericarditis, internal jugular vein thrombosis, and carotid artery erosion may result (Sethi and Stanley 1994; el-Sayed and al Dousary 1996; Sakaguchi et al 1997; Bottin et al 2003). Therefore, coping with deep neck abscess infection remains a challenge to otolaryngologists.

Besides adequate drainage of the abscess, antibiotic therapy is essential to successful treatment. To administer effectively antimicrobial agents to a patient, microbiologic data on the abscess must be obtained. However, it usually takes several days or longer to get the necessary data, and consequently empiric antimicrobial therapy is frequently launched before the definite culture result is available. Different empiric 
antibiotics, which generally were combinations of multiple broad-spectrum antibiotics, have been reported in several studies (Gates 1983; Nagy et al 1997; Sakaguchi et al 1997; Chen et al 1998, 2000; Plaza Mayor et al 2001; McClay et al 2003). The changing trends of deep neck abscess have been described in several studies (Har-El et al 1994; Sethi and Stanley 1994). One crucial issue is probably the resistance to antibiotics. For this reason, it is important to be aware of the coverage rate of different empiric antibiotics.

To address these issues, this investigation conducted a retrospective study analyzing the bacteriology and the antimicrobial susceptibility testing of isolates from patients with deep neck abscess. The effectiveness of different empiric antibiotics on deep neck abscess was statistically analyzed, too.

\section{Patients and methods}

This study retrospectively reviewed the medical records of 981 patients diagnosed with deep neck infection who were admitted to Chang Gung Memorial Hospital in Keelung, Taiwan between April 2001 and October 2006. Diagnostic imaging procedures included computed tomography (CT), ultrasonography (US), and plain radiography. Patients with superficial skin infection or abscess, deep neck infection without evidence of abscess formation, or limited intraoral space infection were excluded. The study enrolled 100 patients. All these patients received abscess drainage via US-guided needle aspiration, or via a surgical incision, or both, with sterilization of intact skin.

Aerobic and anaerobic bacterial cultures were performed for all cases under aseptic field and technique. Patients were treated with empiric antibiotic therapy covering aerobic and anaerobic bacteria. Intravenous broad-spectrum antibiotics were administered before the results of abscess culture became available. Pathogen-directed antibiotic therapy was done when the final results of bacterial cultures were obtained. Abscess specimens were taken and sent by sterile transport swab (Copan, Italy). Then every specimen was inoculated onto 5\% sheep's blood agar, chocolate agar, and eosin-methylene blue (EMB) agar plates for the culture of aerobic and facultative organisms. The plates were incubated at $37{ }^{\circ} \mathrm{C}$ aerobically, under $5 \%$ carbon dioxide, and were examined at 48 and 72 hours. For anaerobes, the material was inoculated onto anaerobic blood agar (CDC) (BBL, USA), whose nutrition base was tryptic soy agar supplemented with yeast extract, vitamin K3, hemin, and 5\% sheep blood. The anaerobic plates were incubated in anaerobic chambers (Concept Plus, Anaerobic Workstation; Ruskinn technology, UK) and examined at 72 and 120 hours. $\beta$-lactamase activity was measured for all isolates with a cefinase disc (chromogenic cephalosporin nitrocefin).

The antimicrobial susceptibility testing was determined by disk diffusion with Mueller-Hinton $(\mathrm{MH})$ agar plates. Additionally, M100-S18 was used for aerobic bacteria. The disks used for gram-positive aerobic bacteria included penicillin, oxacillin, erythromycin, trimethoprim/sulfamethoxazole, clindamycin, chloramphenicol, teicoplanin, and vancomycin. The disks for gram-negative bacteria comprised ampicillin, piperacillin, cefazolin, cefuroxime, ceftriaxone, ceftazidime, imipenem, aztreonam, gentamicin, amikacin trimethoprim/sulfamethoxazole, and ciprofloxacin. In some situations, cefepime, flomoxef, and meropenem were added to the tests for gram-negative aerobes. As for anaerobic pathogens, M11-A7 was the method used for antimicrobial susceptibility testing of anaerobic bacteria. The antibiotic test consisted of penicillin, piperacillin, clindamycin, chloramphenicol, and metronidazole.

Various empiric antibiotics for deep neck infection have been proposed previously (Table 1) (Brondbo et al 1983;

Table I Antibiotics suggested by different authors when awaiting the final bacterial culture results or when there is no purulence for culture ${ }^{a}$

\begin{tabular}{|c|c|c|}
\hline Authors & Antibiotics & $\begin{array}{l}\text { Age group } \\
\text { (number) }\end{array}$ \\
\hline $\begin{array}{l}\text { Sakaguchi et al } \\
\text { (1997) }\end{array}$ & Penicillin derivative and clindamycin & $A(91)$ \\
\hline $\begin{array}{l}\text { Parhiscar and } \\
\text { Har-El (200I) }\end{array}$ & Penicillin G with oxacillin or nafcillin & P\&A (2I0) \\
\hline Gates (1983) & $\begin{array}{l}\text { Penicillin, } \beta \text {-lactamase-resistant drug } \\
\text { such as clindamycin, chloramphenicol, } \\
\text { or gentamicin }\end{array}$ & NA \\
\hline $\begin{array}{l}\text { Chen et al } \\
\text { (1998) }\end{array}$ & Penicillin G, gentamicin and clindamycin & $A(2 \mid 4)$ \\
\hline $\begin{array}{l}\text { Chen et al } \\
(2000)\end{array}$ & Penicillin G, gentamicin and clindamycin & $A(105)$ \\
\hline $\begin{array}{l}\text { Plaza and } \\
\text { Mayor (200I) }\end{array}$ & Cefotaxime and metronidazole & $A(3 I)$ \\
\hline $\begin{array}{l}\text { Simo et al } \\
(1998)\end{array}$ & Flucloxacillin and metronidazole & $P(65)$ \\
\hline $\begin{array}{l}\text { Nagy et al } \\
\text { (1997) }\end{array}$ & Ceftriaxone and clindamycin & NA \\
\hline $\begin{array}{l}\text { McClay et al } \\
(2003)\end{array}$ & Cefuroxime and clindamycin & $P(I I)$ \\
\hline $\begin{array}{l}\text { Sichel et al } \\
(2002)\end{array}$ & Amoxicillin-clavulanic acid & $P(6), A(I)$ \\
\hline $\begin{array}{l}\text { Brondbo et al } \\
\text { (1983) }\end{array}$ & Penicillin G, metronidazole & $A(I)$ \\
\hline
\end{tabular}

Abbreviations: A, adult patient; $P$, pediatric patient; NA, data not available. 
Gates 1983; Nagy et al 1997; Sakaguchi et al 1997; Chen et al 1998, 2000; Simo et al 1998; Parhiscar and Har-El 2001; Plaza Mayor et al 2001; Sichel et al 2002; McClay et al 2003). Five different empiric antibiotics were determined: regimen 1 (penicillin $\mathrm{G}$ and clindamycin and gentamicin), regimen 2 (ceftriaxone and clindamycin), regimen 3 (ceftriaxone and metronidazole), regimen 4 (cefuroxime and clindamycin), and regimen 5 (penicillin and metronidazole), and were analyzed with descriptive statistics (Chi square, degree of freedom, probability value). The cultured bacteria that were susceptible to the antibiotic regimen were defined as sensitive, while those resistant to antibiotics were defined as resistant. Regimen coverage rate is defined as the number of cases with sensitive results divided by the total number of cases.

Descriptive statistics, for example frequency and percentage, were used. Since abscess specimens were all tested with these five regimens, the McNemar test was made to compare the coverage among different antimicrobial regimens. The level of significance was 0.005 due to Bonferrori adjustment (alpha divided by number of pairwise comparison) for multiple comparisons in order to maintain type I error of 0.05 (Bland and Altman 1995).

\section{Results}

The male:female ratio of the 100 enrolled patients was 3:2. Mean age was 49.2 years (range 1-88 years). Age distribution during the study period is shown in Figure 1.

Deep neck abscess involving more than 1 space was found in 29 patients (29\%). Different deep neck space involvement is shown in Table 2. The submandibular space was the most common involvement site (35\%). All 100 patients received abscess drainage by surgical incision or needle aspiration or both. Furthermore, 66 patients $(66 \%)$ received surgical incision and $32(32 \%)$ received needle aspiration. Two cases initially received needle aspiration, but this procedure failed to adequately drain the abscess. Consequently, these two cases received surgical incision. Forty-four patients (44\%) had associated systemic diseases. Diabetes mellitus was the most common (28 patients, $28 \%$ ). Other associated systemic diseases included malignancy ( 6 cases), cerebrovascular accident with bed-ridden status (4 cases), renal failure ( 3 cases), liver cirrhosis ( 2 cases), pulmonary tuberculosis (1 case), and Kawasaki's disease (1 case).

The cause of deep neck abscess was identified in 77 patients $(77 \%)$. The two most common causes were acute oropharyngeal infection (35\%) and odontogenic infection (23\%). Other causes included skin infection, sialolith, sialadenitis, trauma from ingesting foreign body, tuberculosis, and congenital cyst with infection. No definite etiology could be identified in 23 cases (23\%). Notably, all 9 cases of peritonsillar abscess were caused by oropharyngeal infection. Dental infection was not identified as a known cause in cases of retropharyngeal space abscess.

Results of bacterial cultures were available in all 100 patients. Of these, 89 cultures from 89 patients yielded positive results $(89 \%)$ and 11 cases showed no bacterial growth from the cultures. Fifty-one out of 89 positive cultures were polymicrobial $(57.3 \%)$. The remaining 38 cultures grew monomicrobial pathogen $(42.7 \%)$, of which 21 were gram-negative aerobes, 14 were gram-positive aerobes, and 3 were anaerobes (Table 3). A total of 174 isolates were recovered. (1.7 per specimen) (Table 4), and 115 of these were aerobic or facultative organisms (1.2 per specimen). The predominant among these were viridans streptococci, Klebsiella pneumoniae, and Staphylococcus aureus.

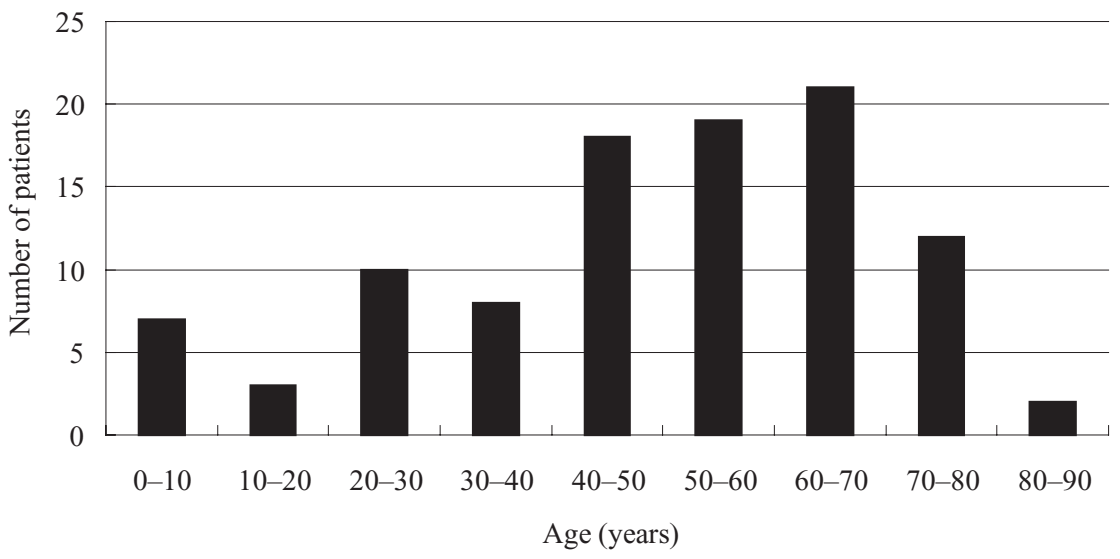

Figure I Age distribution of 100 patients in study with deep neck abscesses. 
Fifty-nine were anaerobic bacteria ( 0.6 per specimen), and the predominant among these were species of Prevotella, Peptostreptococcus, and Bacteroides, including Prevotella intermedia, Peptostreptococcus micros, and unspecified related organisms.

Ten $\beta$-lactamase-producing bacteria (BLPB) were isolated in 10 patients $(11.2 \%)$ (Table 4$)$. These included 7 out of 15 (46.7\%) S. aureus isolates, 2 out of $5(40 \%)$ coagulase-negative staphylococci, and 1 out of $3(33.3 \%)$ Fusobacterium nucleatum isolates.

Judging from the antimicrobial susceptibility tests of 89 patients with positive culture results, the coverage rates of regimen $1,2,3,4$, and 5 were $67.4 \%, 76.4 \%, 70.8 \%, 61.8 \%$, and $16.9 \%$, respectively (Table 5 ). The coverage of regimen 5 was significantly worse than that of the other four regimens $(\mathrm{p}<0.001)$. Regimen 2 was significantly better than regimen $4(\mathrm{p}<0.001)$; regimen 2 was better than regimen 1 $(\mathrm{p}=0.096)$ and regimen $3(\mathrm{p}=0.302)$, but the difference was not statistically significant.

\section{Discussion}

This study illustrates the polymicrobial characteristic of deep neck abscess, which is consistent with previous reports (Gates 1983; Brook 1987; Sethi and Stanley 1994; el-Sayed and al Dousary 1996; Sakaguchi et al 1997; Parhiscar and Har-El 2001; Brook 2002a; McClay et al 2003). When the normal flora of the head and neck region are introduced into a sterile site of the body and results in formation of abscess, the bacteriologic pattern is usually polymicrobial in nature, including aerobes, facultative aerobes, and anaerobes (Brook 2002a). Eighty-nine percent of our bacterial cultures were positive. Moreover, 51 positive cultures were polymicorbial $(57.3 \%)$. Eleven bacterial cultures $(11 \%)$ had no growth

Table 2 Distribution of deep neck abscess location

\begin{tabular}{ll}
\hline Deep neck space & No. of cases (\%) \\
\hline Suprahyoid neck space & \\
Submandibular space & $35(35 \%)$ \\
Parapharyngeal space & $20(20 \%)$ \\
Masticator space & $13(13 \%)$ \\
Peritonsillar space & $9(9 \%)$ \\
Sublingual space & $7(7 \%)$ \\
Parotid space & $3(3 \%)$ \\
Infrahyoid neck space & $16(16 \%)$ \\
Anterior cervical space & $10(10 \%)$ \\
Posterior cervical space & \\
Spaces transversing the whole neck & $13(13 \%)$ \\
Retropharyngeal space & $11(11 \%)$ \\
Carotid space &
\end{tabular}

of any bacteria. Negative culture results may be due to the liberal use of antimicrobial agents prior to admission or intravenous antibiotic treatment before surgical treatment (Sethi and Stanley 1994; el-Sayed and al Dousary 1996; Bottin et al 2003).

Our data revealed that the most common aerobe was viridans streptococci, the second and third ones were $K$. pneumoniae and $S$. aureus. The relatively high incidence of viridans streptococci was consistent with the high rate of cases of dental origin (Parhiscar and Har-El 2001). Similarly, Bottin et al thought that the incidence of Peptostreptococcus sp., viridans streptococci, Streptococcus intermedius, and Streptococcus constellatus was relevant to odontogenic cases of deep neck infection (Bottin et al 2003). In the present study, among the 43 isolates of viridans streptococci, 8 isolates of Peptostreptococcus sp, and 2 isolates of $S$. intermedius, odontogenic cases were the most common (32.1\%), followed by oropharyngeal cases $(28.3 \%)$. The most frequent gram-positive aerobes isolated were composed of staphylococci and streptococci. Similar data were seen in other studies (Brook 1987; Sethi and Stanley 1994; el-Sayed and al Dousary 1996; Sakaguchi et al 1997; Parhiscar and Har-El 2001; Bottin et al 2003). Among the isolates of aerobic organisms, the ratio of gram-positive to gram-negative bacteria was around 3:2 (61.7\%:38.3\%). However, the ratio of gram-negative aerobes was less than $4 \%$ in the study of Parhiscar and Har-El (2001) Gram-positive cocci, such as streptococci and staphylococci, still remain the major aerobic pathogens in deep neck abscess, while the role of gram-negative bacteria emerging as pathogens in deep neck abscess is gaining more and more attention.

In most mucous membranes, anaerobic bacteria are the predominant members of the normal flora, and anaerobes outnumber aerobic and facultative bacteria in ratios ranging from 10:1 to $10,000: 1$, with anaerobic gram-negative bacilli predominating (Tabaqchali 1988; Brook 2002a). The predominant anaerobic bacteria of head and neck infections are of oral flora origin, including Prevotella, Fusobacterium, Bacteroides, and Peptostreptococcus. When natural barriers are broken, these bacteria can cause severe deep neck infection (Fairbanks 2003, p 50). Quite a few of the bacterial infections that occur in the mouth and adjacent areas are believed to be caused by members of the normal commensal flora. Therefore, the role of anaerobic infection should not be neglected. The two most commonly isolated anaerobes in the study were Prevotella and Peptostreptococcus. Other anaerobes included Bacteroides vulgatus and unspecified related organisms, Propionibacterium spp., 
Table 3 Results of bacterial cultures

\begin{aligned} \hline Result & Case no (N = 100) \\ \hline $\begin{array}{c}\text { Positive bacterial culture } \\ \text { Monomicrobial } \\ \text { Gram-positive aerobe }\end{array} & 89 \\$ Gram-negative aerobe & $38(42.70 \%) \\$ Anaerobe & 14 \\ Aerobes only & 3 \\ All gram positive & $5 \mathrm{I}(57.30 \%) \\$ All gram negative & I3 \\ Both gram positive & I \\ and negative & 7 \\ Anaerobes only & 2 \\ Both aerobe(s) and anaerobe(s) & 36 \\ No growth of bacteria & II \end{aligned}

Fusobacterium spp., Veillonella spp., Eubacterium spp. Previous studies identified similar features (Brook 1987; Sethi and Stanley 1994; el-Sayed and al Dousary 1996; Parhiscar and Har-El 2001; Bottin et al 2003).

Forty-one patients in the study had associated systemic diseases, of which diabetes mellitus (28 patients, 58.5\%) was the most common. Patients with underlying diseases, such as diabetes mellitus, uremia, and cancer, are immunocompromised and susceptible to frequent infections. Diabetic patients were reported to be more susceptible to infection by gram-negative rods and staphylococci (el-Sayed and al Dousary 1996). K. pneumoniae was the most common pathogen in $73 \%$ of deep neck infection of diabetes patients in study by Chen et al (2000). In our series, K. pneumoniae was isolated from 18 out of 28 diabetic patients (64.3\%), followed by viridans streptococci $(28.6 \%)$, and $S$. aureus $(14.3 \%)$. It is believed that the virulence of this gram-negative rod is decided by the host's macrophage function (Chen et al 1998, 2000).

Apparently the selection of empiric antibiotics is based on the common pathogens. Antibiotics are mandatory and certainly administered empirically when deep neck abscess is diagnosed initially. As empiric therapy, the antibiotics must "cover" all of the likely pathogens since the infecting organisms have not yet been defined. The microbiology of deep neck infections might change with time, and the resistance to antibiotics might influence the selection of empiric antibiotics. Etiology of deep neck infection could possibly alter in different regions of the world. Intravenous drug abuse, or mandible fracture, which is a frequent cause of deep neck infection in the report by Parhiscar and Har-El (2001) is not commonly seen in Taiwan as a cause of deep neck infection. Antibiotics are mandatory and are used empirically when initially dealing with deep neck abscess. Owing to the polymicrobial nature of deep neck abscess, the use of a single antibiotic without broad-spectrum coverage for aerobes and anaerobes may not have effective treatment response. Administration of more than one kind of antibiotics is usually required as an effective way of treatment.

Penicillin $G$ is the drug of choice for those infections caused by streptococci, and nonpenicillinase-producing staphylococci, which represent the majority of aerobic organisms in deep neck abscess. Gentamicin shows synergism with penicillin in bactericidal activity (Webb et al 1999, p 257), and is frequently used in treating gram-negative microbial infections, especially those due to Pseudomonas, Enterobacter, Klebsiella, or Serratia, which could contribute to the septic process in the immunocompromised hosts. Ototoxicity and nephrotoxicity are the main disadvantages of aminoglycosides. In our study, the Enterobacteriaceae group, including $K$. pneumoniae, K. oxytoca, Enterobacter aerogenes, Morganella morganii, Proteus mirabilis, and Serratia marcescens, were causative pathogens. Consequently, antibiotics against gram-negative bacteria should be chosen as one of the empiric antimicrobial agents. $K$. penumoniae was the second most common aerobe, which was susceptible to cefazolin, gentamicin, and trimethoprim/sulfamethoxazole based on the antimicrobial susceptibility tests. As K. penumoniae is a common pathogen in patients with diabetes mellitus, Chen et al (2000) proposed that empiric antimicrobial regimens must include antibiotics against $K$. pneumoniae in diabetic patients.

Clindamycin is effective against all streptococci, most pneumococci, and most penicillin-resistant (but not methicillin-resistant) staphylococci. In addition, clindamycin is especially useful in against polymicrobial infections of Bacteroides species and other oral anaerobes that are prevalent in chronic tonsillitis and deep neck abscess of dental or oral origin (Brook and Foote 1986). Tabaqchali suggested that treatment of anaerobic infections in the head and neck region should include the use of $\beta$-lactamase-resistant antimicrobials, such as clindamycin or one of the nitroimidazoles with penicillin (Tabaqchali 1988).

Third-generation parenteral cephalosporins have a broad spectrum of activity against enteric gram-negative rods. Compared with first-generation agents, they are less active against gram-positive cocci, such as methicillin-susceptible staphylococci, and anaerobes, but quite active against Hemophilus influenzae, Neisseria, and pneumococci. Ceftriaxone and cefotaxime have desirable activity against streptococci. Besides, ceftriaxone has an excellent gram-negative 
Table 4 Microbiology of 89 patients with deep neck abscess ${ }^{\mathrm{a}}$

Bacteria

Total $(\mathbf{N}=174)$

Aerobic bacteria

Viridans streptococci

Klebsiella penumoniae $\quad 26$

Staphylococcus aureus $\quad 13(7)$

Eikenella corrodens 5

Coagulase-negative staphyloccoci $\quad 5$ (2)

$\beta$-hemolytic Streptococcus non ABD 5

Neisseria sp. 2

Streptococcus intermedium 2

Group D Streptococcus 2

Acinectobacter baumannii I

Escherichia coli I

Enterobacter aerogenes I

Hemophilus influenzae I

Hemophilus parainfluenzae I

Klebsiella oxytoca I

Morganella morganii ।

Proteus mirabilis I

Salmonella enteritidis $D$ ।

Serratia marcescens I

Streptomyces sp. I

Burkholderia cepacia (Pseudomonas cepacia) I

Subtotal aerobes

$115(9)$

Anaerobic bacteria

$\begin{array}{ll}\text { Prevotella sp. } & 10\end{array}$

$\begin{array}{ll}\text { Peptostreptococcus sp. } & 8\end{array}$

$\begin{array}{ll}\text { Prevotella intermedia } & 8\end{array}$

Peptostrepto. micros 7

Bacteroides sp. $\quad 5$

Propionibacterium acnes $\quad 4$

Fusobacterium nucleatum 3

Fusobacterium sp. 3 (I)

Peptostreptococcus magnus 3

Peptostreptococcus anaerobius 2

Veillonella alcalescens 2

Bacteroides vulgatus I

Propionibacterium avidum I

Eubacterium lentum I

GM(+) nonspore forming ।

Subtotal anerobes $59(1)$

Total 174 (10)

${ }^{a}$ Number within parentheses indicates $\beta$-lactamase producers.

spectrum and is active against Hemophilus, most Streptococcus pneumoniae strains and penicillin-resistant Neisseria (Kasper et al 2005, p 798). Streptococci, Enterobacteriaceae group, and staphylococci were the major aerobic pathogens in deep neck abscess, which indicated the administration of ceftriaxone as an appropriate regimen.

Second-generation cephalosporins are generally somewhat less active than first-generation cephalosporins against gram-positive cocci but have a broader spectrum against gram-negative bacilli, such as Enterobacter, Citrobacter, and Morganella. In addition, some provide excellent activity against $H$. influenzae, and others have added activity against anaerobic bacteria, particularly Bacteroides fragilis (Gantz et al 1999). Cefuroxime, a second-generation cephalosporin, is highly active against gram-positive cocci, but more importantly, it is effective against $H$. influenzae and Moraxella catarrhalis, including the ampicillin-resistant strains (Bailey 2001, p 48-55).

Metronidazole is active against almost all obligate anaerobes, including the genera Peptostreptococcus, Bacteroides, Prevotella, Fusobacterium, and Clostridium, but it is not active against aerobes. When administered as an empiric antibiotic for deep neck abscess, metronidazole should be combined with antibiotics effective against aerobes to achieve broad coverage.

Ten of the 174 isolates were $\beta$-lactamase-producing strains $(5.8 \%)$. These strains were isolated from 10 patients $(10 \%)$, and 7 were $S$. aureus, 2 were coagulase-negative staphylococcus, and 1 was $F$. nucleatum. Resistance to $\beta$-lactam antibiotics is considered closely related to the emergence of BLPB (Kuriyama et al 2000). These organisms can survive penicillin therapy and may protect penicillin-susceptible bacteria from penicillin by releasing the enzyme into the abscess cavity (Brook 1987). Resistance of S. aureus and Bacteroides spp. to penicillin has been recognized for over 2 decades. However, in recent years, a growing number of anaerobic gram-negative bacilli, Prevotella and Fusobacterium spp. in particular, have been observed to produce the enzyme (Brook 2002b).

Production of $\beta$-lactamase would protect not only the BLPB but also the $\beta$-lactamase nonproducing bacteria from $\beta$-lactam antibiotics. BLPB could be a crucial issue in deep neck abscesses as they could be one of the polymicrobial pathogens in abscesses. Rapid emergence of aerobic and anaerobic BLPB after 1 course of penicillin therapy in children has been demonstrated (Brook and Gober 1984). Besides, Kuriyama et al found an interesting correlation between the incidence of BLPB and the duration of $\beta$-lactam administration in past treatment of orofacial odnotogenic infections (Kuriyama et al 2000). The growing resistance of the bacteria to $\beta$-lactam regimens limits the usefulness of this kind of drug. Accordingly, the appearance of resistant bacteria should warrant the administration of appropriate antimicrobial agents that are effective against aerobic and anaerobic BLPB (eg, clindamycin, imipenem, combination of metronidazole and a macrolide, or amoxicillin and clavulanic acid). BLPB should be considered especially if the infection still persists despite abscess drainage (Brook 1987). 
Table 5 The coverage rate between different antimicrobial regimens upon the results of aerobic and anaerobic bacterial cultures of 89 patients with positive culture results

\begin{tabular}{lllll}
\hline Code name & Antibiotics & $\begin{array}{l}\text { No. of cases whose } \\
\text { cultures were sensitive }\end{array}$ & $\begin{array}{l}\text { No. of cases whose } \\
\text { cultures were resistant }\end{array}$ & Coverage rate (\%) \\
\hline Regimen I & Penicillin, gentamicin, clindamycin & 60 & 29 & $67.42 \%$ \\
Regimen 2 & Ceftriaxone, clindamycin & 68 & 21 & $76.40 \%$ \\
Regimen 3 & Ceftriaxone, metronidazole & 63 & 26 & $70.79 \%$ \\
Regimen 4 & Cefuroxime, clindamycin & 55 & 34 & $61.80 \%$ \\
Regimen 5 & Penicillin G, metronidazole & 15 & 74 & $16.85 \%$ \\
\hline
\end{tabular}

The coverage rate of different combinations of empiric antibiotics, as suggested by previous published articles (Gates 1983; Nagy et al 1997; Sakaguchi et al 1997; Chen et al 1998, 2000; Plaza Mayor et al 2001; McClay et al 2003) was compared based on the antimicrobial susceptibility of all cases in this study (Table 5). Regimen 5, penicillin and metronidazole, was less efficacious than the other four regimens $(\mathrm{p}<0.001)$ because penicillin and metronidazole could not adequately cover the gram-negative organisms, reducing the utility of the regimen. Among regimens 1-4, regimen 2 was found to be superior to regimens 1,3 , and 4 . Regimens 2 and 4 differed significantly $(\mathrm{p}<0.001)$. Based on the drug sensitivity tests of the bacterial culture results, regimen 2 was more effective than regimen 4 by 13 patients. The cultured bacteria of these 13 patients were all susceptible to regimen 2 and resistant to regimen 4 . The pathogens included viridans streptococci (11 isolates), S. aureus (1), S. intermedius (1), $\beta$-hemolytic Streptococcus group non-ABD (1), Salmonella enteritidis D (1), and Prevotella spp. (1), which were sensitive to ceftriaxone but not cefuroxime. The coverage rate of regimen 2 was better than regimens 1 and 3 but not significantly.

Surgical drainage is of paramount importance in the treatment of deep neck abscess. Additionally, antimicrobial therapy effective against the aerobic and anaerobic bacteria is essential for achieving complete resolution of the abscess (Simo et al 1998; Brook 2002a). According to the culture presented in this study, cases with only one single pathogen occupied $42.7 \%$ (38 cases) (Table 2). Gram-positive aerobes were isolated in 14 cases, gram-negative aerobes in 21 cases, and anaerobes in 3 cases. In the 51 cases (57.3\%) with polymicrobial infection, 13 cases were aerobes only, 2 cases were anaerobes only, and the remaining 36 cases were mixed aerobic and anaerobic infection. The variability of microbial infection was clearly delineated. Even in the cases involving monomicrobial infection, it was impossible to find a single effective antibiotic before the culture results were available. Therefore, combination of antimicrobial agents covering the gram-positive aerobes, gram-negative aerobes, and anaerobes should be chosen as empiric antibiotic treatment. Specific antimicrobial therapy can be selected after obtaining the culture results.

\section{Conclusion}

Polymicrobial infection accounts for most deep neck abscess. Gram-positive aerobes, gram-negative aerobes and anaerobes are all common pathogens. Variability of pathogens is found either in polymicrobial or monomicrobial infection, which indicates the combination of antimicrobial agents covering both aerobes and anaerobes as an appropriate empiric antibiotic treatment. Ceftriaxone and clindamycin, ceftriaxone and metronidazole, or penicillin $\mathrm{G}$ and gentamicin and clindamycin could be good candidates for empiric antibiotics for treating deep neck abscess in this study.

\section{Acknowledgments}

Source of funding: Supported by grants from Chang Gung Research Program CMRPG2014.

Institution of the work done: Chang Gung Memorial Hospital, Keelung, Taiwan.

\section{Disclosures}

None of the authors has any conflicts of interest to disclose.

\section{References}

Bailey B. 2001. Head and Neck Surgery-Otolaryngolgoy Phiadelphia: Lippincott Williams and Wilkins.

Bland JM, Altman DG. 1995. Multiple significance tests: the Bonferroni method. BMJ, 310:170.

Bottin R, Marioni G, Rinaldi R, et al. 2003. Deep neck infection: a presentday complication. A retrospective review of 83 cases (1998-2001). Eur Arch Otorhinolaryngol, 260:576-9.

Brondbo K, Rubin A, Chapnik JS, et al. 1983. Ludwig's angina following dental extraction as a cause of necrotizing mediastinitis. J Otolaryngol, 12:50-2.

Brook I, Gober AE. 1984. Emergence of beta-lactamase-producing aerobic and anaerobic bacteria in the oropharynx of children following penicillin chemotherapy. Clin Pediatr (Phila), 23:338-41.

Brook I, Foote PA Jr. 1986. Comparison of the microbiology of recurrent tonsillitis between children and adults. Laryngoscope, 96:1385-8.

Brook I. 1987. Microbiology of abscesses of the head and neck in children. Ann Otol Rhinol Laryngol, 96:429-33. 
Brook I. 2002a. Microbiology of polymicrobial abscesses and implications for therapy. J Antimicrob Chemother, 50:805-10.

Brook I. 2002b. Aerobic and anaerobic microbiology of suppurative sialadenitis. J Med Microbiol, 51:526-9.

Chen MK, Wen YS, Chang CC, et al. 1998. Predisposing factors of lifethreatening deep neck infection: logistic regression analysis of 214 cases. J Otolaryngol, 27:141-4.

Chen MK, Wen YS, Chang CC, et al. 2000. Deep neck infections in diabetic patients. Am J Otolaryngol, 21:169-73.

el-Sayed Y, al Dousary S. 1996. Deep-neck space abscesses. J Otolaryngol, 25:227-33.

Fairbanks D. 2003. Pocket guide to antimicrobial therapy in otolaryngology head and neck surgery. Virginia: The American Academy of Otolaryngology - Head and Neck Surgery Foundation, Inc.

Gantz N, Brown R, Berk S, et al. 1999. Manual of Clinical Problems in Infectious Diseases. Philadelphia: Lippincott Williams and Wilkins.

Gates GA. 1983. Deep neck infection. Am J Otolaryngol, 4:420-1.

Har-El G, Aroesty JH, Shaha A, et al. 1994. Changing trends in deep neck abscess. A retrospective study of 110 patients. Oral Surg Oral Med Oral Pathol, 77:446-50.

Kasper D, Braunwald E, Hauser S, et al. 2005. Harrison's Principles of Internal Medicine. New York: McGraw-Hill.

Kuriyama T, Nakagawa K, Karasawa T, et al. 2000. Past administration of beta-lactam antibiotics and increase in the emergence of beta-lactamaseproducing bacteria in patients with orofacial odontogenic infections. Oral Surg Oral Med Oral Pathol Oral Radiol Endod, 89:186-92.
McClay JE, Murray AD, Booth T. 2003. Intravenous antibiotic therapy for deep neck abscesses defined by computed tomography. Arch Otolaryngol Head Neck Surg, 129:1207-12.

Nagy M, Pizzuto M, Backstrom J, et al. 1997. Deep neck infections in children: a new approach to diagnosis and treatment. Laryngoscope, 107:1627-34.

Parhiscar A, Har-El G. 2001. Deep neck abscess: a retrospective review of 210 cases. Ann Otol Rhinol Laryngol, 110:1051-4.

Plaza Mayor G, Martinez-San Millan J, Martinez-Vidal A. 2001. Is conservative treatment of deep neck space infections appropriate? Head Neck, 23:126-33.

Sakaguchi M, Sato S, Ishiyama T, et al. 1997. Characterization and management of deep neck infections. Int J Oral Maxillofac Surg, 26:131-4.

Sethi DS, Stanley RE. 1994. Deep neck abscesses-changing trends. $J$ Laryngol Otol, 108:138-43.

Sichel JY, Dano I, Hocwald E, et al. 2002. Nonsurgical management of parapharyngeal space infections: a prospective study. Laryngoscope, 112:906-10.

Simo R, Hartley C, Rapado F, et al. 1998. Microbiology and antibiotic treatment of head and neck abscesses in children. Clin Otolaryngol Allied Sci, 23:164-8.

Tabaqchali S. 1988. Anaerobic infections in the head and neck region. Scand J Infect Dis Suppl, 57:24-34.

Webb A, Shapiro M, Singer M, et al. 1999. Oxford Textbook of Critical Care. New York: Oxford University Press. 